\title{
B physics at the HL-LHC with the upgraded CMS detector
}

\author{
Sara Fiorendi* on behalf of the CMS Collaboration \\ INFN and University of Milano - Bicocca
}

E-mail: sara.fiorendiecern.ch

The high luminosity LHC (HL-LHC) run, which is due to start in 2026, is expected to deliver an integrated luminosity of approximately $3000 \mathrm{fb}^{-1}$ at a $p p$ center of mass value of $14 \mathrm{TeV}$. Significant upgrades of the CMS detector are foreseen to withstand the highly-demanding operating conditions and to fully exploit the delivered luminosity. More precise investigations of rare decays in the flavour sector will be possible thanks to the large collected data sample. The perspectives for the measurements of the $B_{s}^{0} \rightarrow \mu^{+} \mu^{-}$and $B^{0} \rightarrow \mu^{+} \mu^{-}$branching fractions are reported, together with the projections for the exclusion limit on the $\tau \rightarrow 3 \mu$ branching fraction.

The International Conference on B-Physics at Frontier Machines - BEAUTY2018

6-11 May, 2018

La Biodola, Elba Island, Italy

\footnotetext{
${ }^{*}$ Speaker.
} 
The High Luminosity LHC (HL-LHC) program is scheduled to start in 2026, with the goal of delivering an integrated luminosity of $3000 \mathrm{fb}^{-1}$ at $\sqrt{s}=14 \mathrm{TeV}$ in 10 years of operations [1]. The design instantaneous luminosity of the accelerator is $5 \times 10^{34} \mathrm{~cm}^{-2} \mathrm{~s}^{-1}$ and could be pushed to $7.5 \times 10^{34} \mathrm{~cm}^{-2} \mathrm{~s}^{-1}$, leading to substantially increased simultaneous interactions (140-200 pileup events) with respect to the current operating conditions.

In order to maintain excellent physics performance, CMS plans to replace and upgrade some of its subdetectors for the Phase-II [2]. The current tracker will be replaced by a new detector featuring higher radiation tolerance and enhanced coverage [3]. The inner region will be instrumented with thin silicon pixel sensors, arranged in four cylindrical layers in the barrel region, and eight small plus four large disc-like structures in each forward direction, extending the acceptance up to $|\eta| \approx$ 4. The outer tracker will be composed by six cylindrical barrel layers plus five endcap disks of silicon modules with strip and macro-pixel sensors. The performance of the track reconstruction in terms of track finding efficiency, track fake rate and resolution of the estimated track parameters is shown in Fig. 1 for single muons and tracks from $t \bar{t}$ events.
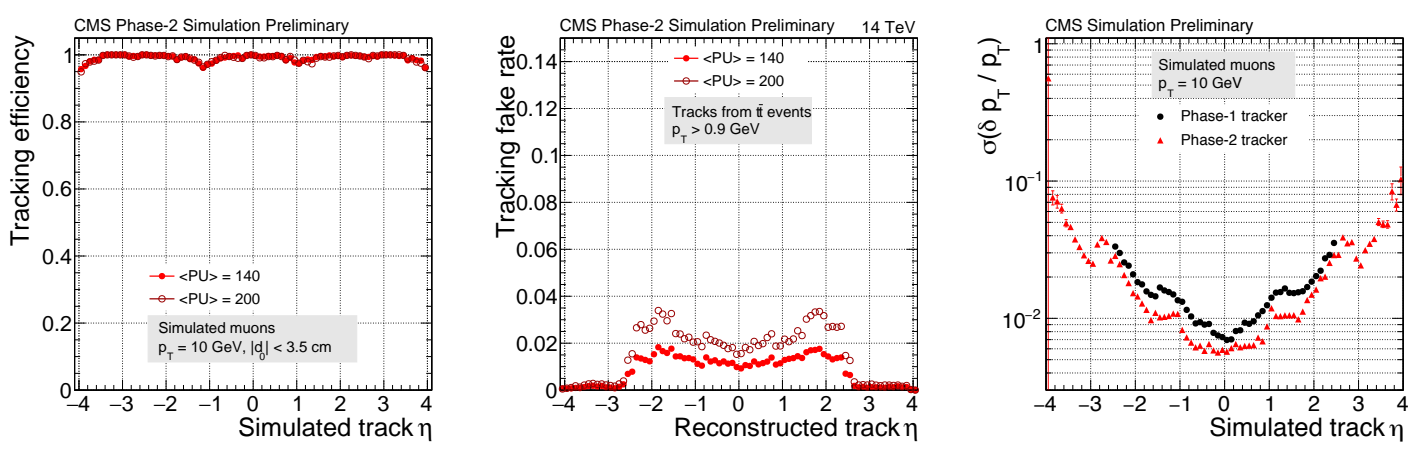

Figure 1: (Left) Tracking efficiency as a function of the pseudorapidity for single muons with $\mathrm{p}_{\mathrm{T}}$ equal to $10 \mathrm{GeV}$ and transverse impact parameter $\left|d_{0}\right|$ below $3.5 \mathrm{~cm}$, for two different pileup scenarios. (Centre) Tracking fake rate as a function of the pseudorapidity for $t \bar{t}$ events in two different pileup scenarios, for tracks with $\mathrm{p}_{\mathrm{T}}$ greater than $0.9 \mathrm{GeV}$. (Right) Relative transverse momentum resolution as a function of the pseudorapidity for the Phase-I (black dots) and the upgraded (red triangles) tracker, using single isolated muons with a transverse momentum of $10 \mathrm{GeV}$.

The outer tracker will provide tracking information to the level-1 (L1) hardware trigger for tracks above a certain $\mathrm{p}_{\mathrm{T}}$ threshold. The on-detector data reduction will be possible thanks to " $\mathrm{p}_{\mathrm{T}}$ modules" [3], which, by correlating charged particle hit positions in their top and bottom sensors, are capable of performing a local measurement of the track transverse momentum, and rejecting signals from particles below the threshold. The usage of tracking information in the hardware trigger will improve the transverse momentum and longitudinal position resolution of various objects. Fig. 2 shows the simulated L1 trigger tracking efficiency for prompt muons and electrons for $t \bar{t}$ events in a scenario with 200 pileup events on average; the resolution on the longitudinal impact parameter $\mathrm{z}_{0}$ of muons with $\mathrm{p}_{\mathrm{T}}>10 \mathrm{GeV}$ is also shown for various average pileup scenarios.

The improved performance of the hardware trigger will allow to access final states that are currently not covered by the existing triggers. As a show case, the decay $B^{0} \rightarrow \phi \phi \rightarrow 4 K$ is presented [3]. This decay is sensitive to beyond standard model contributions, and suitable to determine 

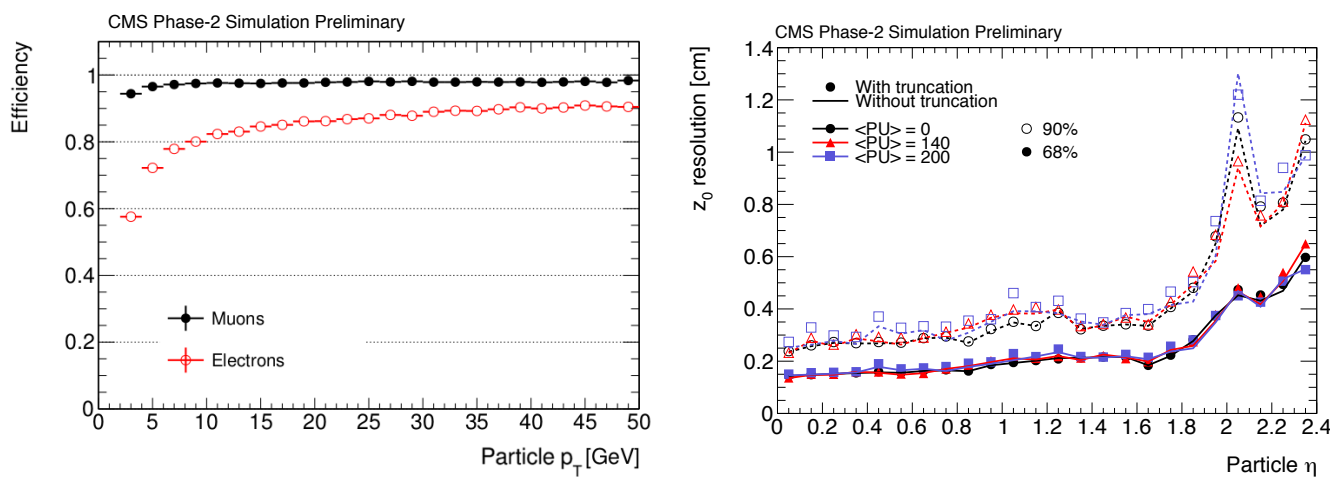

Figure 2: (Left) L1 tracking efficiency versus generated particle $\mathrm{p}_{\mathrm{T}}$ for $t \bar{t}$ events in a scenario with 200 pileup events on average. Results for muons (electrons) are shown as filled black (open red) circles. (Right) Relative $\mathrm{z}_{0}$ resolution versus $|\eta|$ for muons in $t \bar{t}$ events with zero (black dots), 140 (red triangles), and 200 (blue squares) pileup events on average. The effect of the limited number of objects being processed by the trigger algorithm (called truncation) is also displayed.

the $\mathrm{CP}$ violating phase in the $\mathrm{CKM}$ matrix. Thanks to the tracking information, the identification of pairs of oppositely charged tracks originating from the same vertex, then combined into a $\mathrm{B}^{0}$ candidate, will be possible already at L1 and topological selections can be applied to achieve a sustainable rate. The distributions of the invariant mass of the $\phi \phi$ and $\mathrm{B}^{0}$ candidates are presented in Fig. 3 for signal events at the trigger and offline reconstruction levels, and for background events at the trigger level, as obtained from simulated events with 200 simultaneous interactions on average.
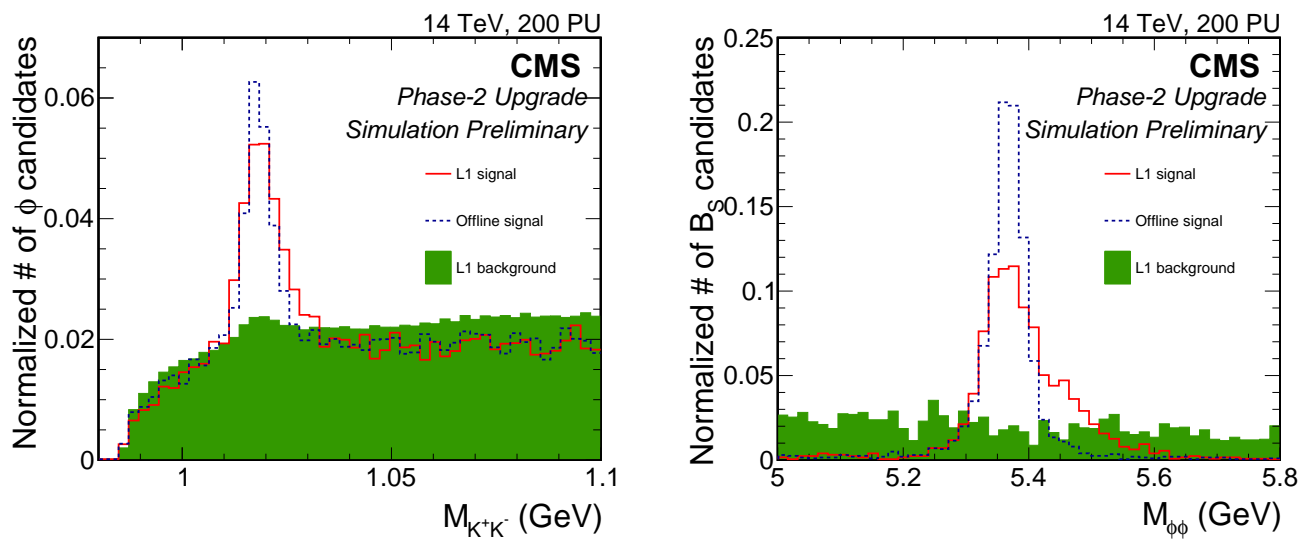

Figure 3: (Left) Invariant mass distribution of track pairs with opposite charges, separation along the beam axis $\Delta z<1 \mathrm{~cm}$, distance in the plane perpendicular to the beam axis $\Delta x y<1 \mathrm{~cm}$, track $\mathrm{p}_{\mathrm{T}}>2 \mathrm{GeV}$, and assuming the kaon mass. The distributions are normalized to unit area. (Right) Invariant mass distribution of all the $\phi$-pairs with $\Delta z$ ( $\phi$-pair $)<1 \mathrm{~cm}, \Delta x y(\phi$-pair $)<1 \mathrm{~cm}, 0.2<\Delta R(\phi$-pair $)<1, \Delta R\left(K^{+}, K^{-}\right)<0.12$, where $\Delta R$ is the distance in the $\eta-\phi$ plane. The distributions are normalized to unit area.

In addition, the muon system will undergo an upgrade campaign: the existing muon detectors and the associated electronics will be ameliorated, in order to ensure good performance and radia- 
tion tolerance; the forward region $(1.6<|\eta|<2.4)$ will be equipped with supplementary resistive plate chambers and gas electron multiplier (GEM) chambers, to increase redundancy and improve trigger and reconstruction capabilities; finally, the pseudorapidity coverage will be extended up to $|\eta|=2.8$ thanks to the addition of a forward muon GEM chamber (ME0)[4]. The muon trigger rate of the upgraded L1 standalone muon trigger is compared to that of the Phase-I detector in the left plot of Fig. 4. The rightmost plot shows the benefit in momentum resolution of the combination of the upgraded muon detector with the new L1 tracking trigger, in terms of the $\mathrm{p}_{\mathrm{T}}$ turn-on curve of such a combined trigger.
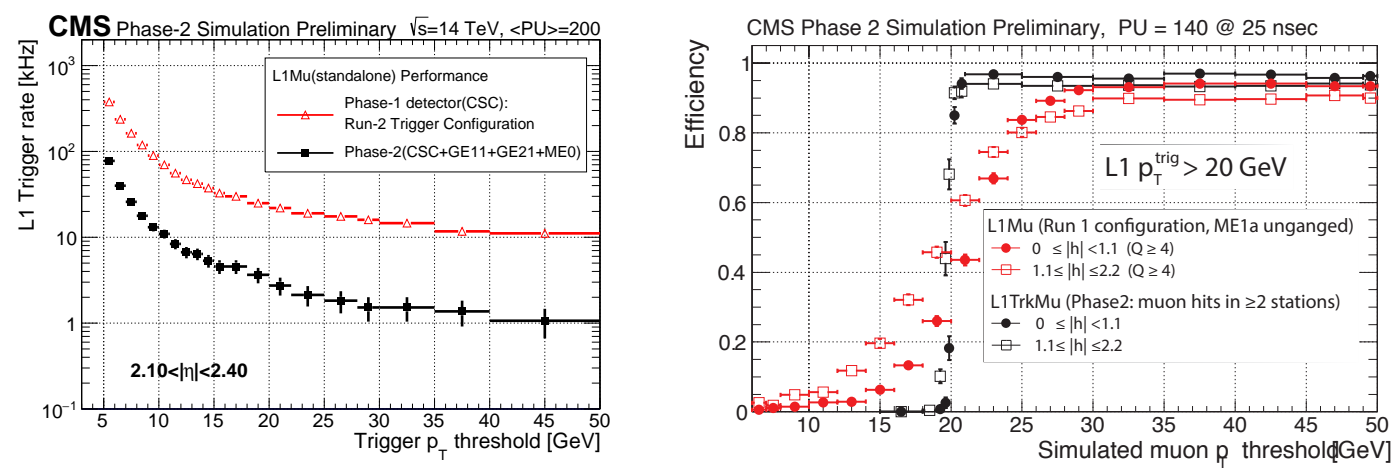

Figure 4: (Left) L1 standalone muon trigger rate for the prompt muon algorithm, with and without GEM chambers included, as a function of the true muon $\mathrm{p}_{\mathrm{T}}$ in the region $2.1<|\eta|<2.4$. (Right) Efficiency of the combined L1Trk+L1Mu trigger as a function of true muon $\mathrm{p}_{\mathrm{T}}$. Two pseudorapidity ranges are shown. For triggers based on standalone $\mathrm{L} 1$ muons, the quality cut $(\mathrm{Q} \geq 4)$ that was used during Run 1 is applied.

\section{Perspectives for $B_{(s)} \rightarrow \mu^{+} \mu^{-}$}

The decays $B_{s}^{0} \rightarrow \mu^{+} \mu^{-}$and $B^{0} \rightarrow \mu^{+} \mu^{-}$are highly suppressed in the standard model (SM), since they can only proceed through flavour changing neutral current processes, forbidden at tree level. The SM predictions are $(3.56 \pm 0.30) \times 10^{-9}$ and $(1.07 \pm 0.10) \times 10^{-10}$ for the $\mathrm{B}_{\mathrm{s}}^{0}$ and $\mathrm{B}^{0}$ decays respectively [9]. The branching fractions are enhanced or further suppressed by many extensions of the SM [10,11,12,13], making these decays an excellent probe for physics beyond the SM. The current experimental results from the ATLAS, LHCb, and CMS Collaborations [5, 6, 7, 8] are all in agreement with the SM expectations, though still statistically limited.

An estimate of the analysis performance with the upgraded CMS detector is presented. The predictions [14] are based on the CMS analysis of the Run I dataset, described in [5, 6]. Possible improvements in the analysis strategy have not been considered in the extrapolation. The analysis sensitivity is determined in two scenarios: the 2019 Phase-I detector with an aging corresponding to $1000 \mathrm{fb}^{-1}$ of integrated luminosity, and the Phase-II upgraded detector. Results are presented for various integrated luminosities: 100 and $300 \mathrm{fb}^{-1}$ for the Phase-I scenario, and $3000 \mathrm{fb}^{-1}$ for the Phase-II scenario. The result at $300 \mathrm{fb}^{-1}$ for the Phase-I scenario is given also for muons in the barrel only to allow for a better comparison with the Phase-II projection. 
Pseudo-experiments which start from the baseline Run 1 PDFs for the signals and the backgrounds are used. In addition, the following assumptions and results from studies on the simulation are included:

- $\mathrm{SM}$ production cross sections and branching fractions are assumed for $\mathrm{B}^{0}$ and $\mathrm{B}_{\mathrm{s}}^{0}$.

- A L1 trigger algorithm requiring two opposite charge muons with transverse momentum greater than $3 \mathrm{GeV},|\eta|<2$ and originating from the same primary vertex is used. Thanks to the $\mathrm{L} 1$ track trigger, the invariant mass resolution is measured to be around $70 \mathrm{MeV}$ both for the $\mathrm{B}_{\mathrm{s}}^{0}$ and the $\mathrm{B}^{0}$, allowing to apply a stringent mass requirement already at the L1 trigger level. Studies on minimum bias samples have shown that the rate of this $\mathrm{L} 1$ trigger corresponds to a very small fraction of the available bandwidth.

- Pileup effects: the large number of primary vertices will impact discriminating variables, such as isolation, and entails a less efficient muon reconstruction due to the tighter cuts needed to maintain a low level of fake tracks (an efficiency loss of $2.5 \%$ per each muon is estimated). Including these effects, the analysis efficiency decreases by $35 \%$ for signal and $30 \%$ for backgrounds.

- Dimuon mass resolution: the improved momentum resolution of tracks for Phase-II translates to a $40 \%$ improvement in the dimuon mass resolution for $|\eta|<1.0$, and a $25 \%$ gain in the significance of the separation between the $\mathrm{B}^{0}$ and $\mathrm{B}_{\mathrm{s}}^{0}$ mass peaks, which translates in an improved background rejection and reduced cross-feed from the $\mathrm{B}_{\mathrm{s}}^{0}$ to the $\mathrm{B}^{0}$ signal region. The dimuon mass resolution and the expected dimuon invariant mass distribution are shown in Fig.5.

- Systematic uncertainties: the systematic uncertainty due to the $f_{s} / f_{u}$ ratio is estimated to be reduced from $9 \%$ to $5 \%$ in the Phase-II scenario. The uncertainty on the yields of the normalization channel is evaluated to be reduced to $3 \%$. The uncertainty due to the peaking and semileptonic backgrounds will be reduced to 10 and $20 \%$ respectively, thanks to a more precise determination of the misidentification rate.

The final estimate of the analysis performance is summarized in Table 1 in terms of uncertainties on the $\mathrm{B}_{\mathrm{s}}^{0}$ and $\mathrm{B}^{0}$ branching fractions and on their ratio, and $\mathrm{B}^{0}$ detection sensitivity, for different values of the integrated luminosity. Thanks to the improved performance of the Phase-II detector, the observation of $B^{0} \rightarrow \mu^{+} \mu^{-}$with a significance larger than $5 \sigma$ will be possible using $3000 \mathrm{fb}^{-1}$ of data, and $\mathscr{B}\left(B^{0} \rightarrow \mu^{+} \mu^{-}\right)$and its ratio to $\mathscr{B}\left(B_{s}^{0} \rightarrow \mu^{+} \mu^{-}\right)$could be measured with precisions of $18 \%$ and $21 \%$, respectively.

\section{Search for the lepton flavour violating decay $\tau \rightarrow 3 \mu$}

The $\tau \rightarrow 3 \mu$ decay is a lepton flavour violating process, which has a predicted branching fraction of $10^{-14}$ in the SM [16]. Several beyond standard model predictions foresee enhancements of the branching ratio by many order of magnitudes. The currently best experimental upper limit has been set by the Belle Collaboration to be $\mathscr{B}(\tau \rightarrow 3 \mu)<2.1 \times 10^{-8}$ at $90 \%$ CL [17]. 


\begin{tabular}{|c|c|c|c|c|}
\hline $\mathscr{L}\left[\mathrm{fb}^{-1}\right]$ & $\delta \mathscr{B}\left(B_{s}^{0} \rightarrow \mu^{+} \mu^{-}\right)$ & $\delta \mathscr{B}\left(B^{0} \rightarrow \mu^{+} \mu^{-}\right)$ & $\mathrm{B}_{\mathrm{d}}^{0}$ sign. & $\delta \frac{\mathscr{B}\left(B^{0} \rightarrow \mu^{+} \mu^{-}\right)}{\mathscr{B}\left(B_{s}^{0} \rightarrow \mu^{+} \mu^{-}\right)}$ \\
\hline 100 & $14 \%$ & $63 \%$ & $0.6-2.5 \sigma$ & $66 \%$ \\
300 & $12 \%$ & $41 \%$ & $1.5-3.5 \sigma$ & $43 \%$ \\
300 (barrel) & $13 \%$ & $48 \%$ & $1.2-3.3 \sigma$ & $50 \%$ \\
3000 (barrel) & $11 \%$ & $18 \%$ & $5.6-8.0 \sigma$ & $21 \%$ \\
\hline
\end{tabular}

Table 1: The estimated analysis sensitivity from pseudo-experiments for different integrated luminosities. Results up to $300 \mathrm{fb}^{-1}$ are for the Phase-I scenario, whereas the result for $3000 \mathrm{fb}^{-1}$ is for the Phase-II.
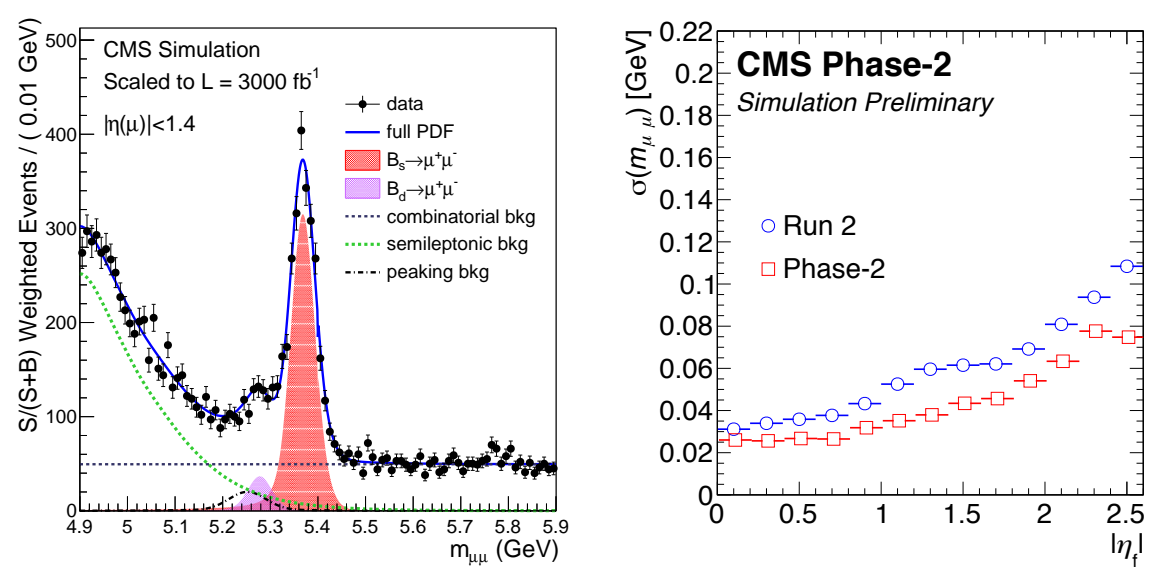

Figure 5: (Left) Projections of the mass fits to $3000 \mathrm{fb}^{-1}$ of integrated luminosity, assuming the expected performances of Phase-II detectors. (Right) Mass resolution as a function of $|\eta|$ for $B_{s}^{0} \rightarrow \mu^{+} \mu^{-}$in Run 2 (blue) and Phase-II (red).

The main source of $\tau$ leptons at the LHC is constituted by $D_{s} \rightarrow \tau v_{\tau}$ decays. However, such $\tau$ leptons and, hence, muons from $\tau \rightarrow 3 \mu$ decays, have very low momenta and are significantly boosted in the forward direction, so that only in $1.3 \%$ of signal events all the three muons lie within the acceptance of the current detector. The study of this decay mode with the CMS Phase-II upgraded detector will benefit from the extended pseudorapidity coverage of the muon system and the improved trigger resolution at low $\mathrm{p}_{\mathrm{T}}$. The available projections of the analysis performance [4] are based on a simulation that includes the new GEM chamber in the very forward region, but does not fully implement the foreseen improvements in the muon reconstruction.

Events are split in two categories: Category 1 contains those events with the three muons within the Phase-I CMS detector acceptance, while Category 2 includes the additional events that would be recorded thanks to the ME0 chamber. A L1 trigger algorithm which requires three muons with invariant mass lower than $3 \mathrm{GeV}$ and further specialized for the two categories is employed, together with a dedicated offline muon identification strategy targeting low momentum muons. The signal-from-noise discrimination is performed using a discriminant built on the product of ratios of 1D signal and background probability density functions for more than a dozen observables describing the three-muon vertex and the event kinematic and topological properties. The number of signal 
events is extracted from a fit to the three muon mass in the two categories, and the $D_{s} \rightarrow \phi(\mu \mu) \pi$ decay mode is used to derive the signal normalization from data and convert the signal yield into a branching fraction measurement. Systematic uncertainties related to the $D$ meson production cross section and background shape have been taken into account. The projected exclusion limit on $\mathscr{B}(\tau \rightarrow 3 \mu)$ is $3.7 \times 10^{-9}$ at $90 \% \mathrm{CL}$ using the full HL-LHC dataset, and the $5 \sigma$-observation sensitivity is $1.1 \times 10^{-8}$.

\section{Summary}

The High Luminosity phase of the LHC will extend the physics reach of the CMS detector, allowing the access to very rare decay modes in the flavour sector. Some selected analyses have been presented, together with a brief overview of the planned detector upgrades, which will be essential to maintain the excellent performances on object reconstruction and achieve the physics goals of CMS at HL-LHC.

\section{References}

[1] G. Apollinari, I. Béjar Alonso, O. Brüning, P. Fessia, M. Lamont, L. Rossi and L. Tavian, High-Luminosity Large Hadron Collider (HL-LHC) : Technical Design Report V. 0.1, CERN Yellow Report CERN 2017-007-M [doi:10.23731/CYRM-2017-004].

[2] D. Contardo, M. Klute, J. Mans, L. Silvestris and J. Butler, Technical Proposal for the Phase-II Upgrade of the CMS Detector CERN-LHCC-2015-010 [https://cds.cern.ch/record/2020886].

[3] CMS Collaboration, The Phase-2 Upgrade of the CMS Tracker, CERN-LHCC-2017-009. CMS-TDR-014 [https://cds.cern.ch/record/2272264].

[4] CMS Collaboration, The Phase-2 Upgrade of the CMS Muon Detectors, CERN-LHCC-2017-012. CMS-TDR-016 [http://cds.cern.ch/record/2283189].

[5] S. Chatrchyan et al. [CMS Collaboration], Measurement of the $B_{s}^{0} \rightarrow \mu^{+} \mu^{-}$branching fraction and search for $B^{0} \rightarrow \mu^{+} \mu^{-}$with the CMS Experiment, Phys. Rev. Lett. 111 (2013) 101804 [hep-ex/1307.5025].

[6] V. Khachatryan et al. [CMS and LHCb Collaborations], Observation of the rare $B_{s}^{0} \rightarrow \mu^{+} \mu^{-}$decay from the combined analysis of CMS and LHCb data, Nature 522 (2015) 68 [hep-ex/1411.4413].

[7] M. Aaboud et al. [ATLAS Collaboration], Study of the rare decays of $B_{s}^{0}$ and $B^{0}$ into muon pairs from data collected during the LHC Run 1 with the ATLAS detector, Eur. Phys. J. C 76, no. 9, 513 (2016) [hep-ex/1604.04263].

[8] R. Aaij et al. [LHCb Collaboration], Measurement of the $B_{s}^{0} \rightarrow \mu^{+} \mu^{-}$branching fraction and effective lifetime and search for $B^{0} \rightarrow \mu^{+} \mu^{-}$decay, Phys. Rev. Lett. 118 (2017) no.19, 191801 [hep-ex/1703.05747].

[9] A. J. Buras, J. Girrbach, D. Guadagnoli and G. Isidori, On the Standard Model prediction for $\mathscr{B}\left(B_{s, d} \rightarrow \mu^{+} \mu^{-}\right)$, Eur. Phys. J. C 72 (2012) 2172 [hep-ph/1208.0934].

[10] J. K. Parry, Lepton flavor violating Higgs boson decays, $\tau \rightarrow \mu \gamma$ and $B_{s} \rightarrow \mu^{+} \mu^{-}$in the constrained $M S S M+N R$ with large tan beta, Nucl. Phys. B 760 (2007) 38 [hep-ph/0510305].

[11] S. R. Choudhury, A. S. Cornell, N. Gaur and G. C. Joshi, Signatures of new physics in dileptonic B-decays, Int. J. Mod. Phys. A 21 (2006) 2617 [hep-ph/0504193]. 
[12] J. R. Ellis, J. S. Lee and A. Pilaftsis, B-Meson Observables in the Maximally CP-Violating MSSM with Minimal Flavour Violation, Phys. Rev. D 76 (2007) 115011 [hep-ph/0708.2079].

[13] J. R. Ellis, K. A. Olive, Y. Santoso and V. C. Spanos, On $B_{s} \rightarrow \mu^{+} \mu^{-}$and cold dark matter scattering in the MSSM with non-universal Higgs masses, JHEP 0605 (2006) 063 [hep-ph/0603136].

[14] CMS Collaboration, CMS reach in $B_{(s)}$ to dimuon and $B$ to dimuon branching fractions for the new LHC runs, CMS-PAS-FTR-13-022 [http://cds.cern.ch/record/1605250].

[15] X. Y. Pham, Lepton flavor changing in neutrinoless tau decays, Eur. Phys. J. C 8 (1999) 513 [hep-ph/9810484].

[16] K. Hayasaka et al., Search for Lepton Flavor Violating Tau Decays into Three Leptons with 719 Million Produced Tau+Tau- Pairs, Phys. Lett. B 687 (2010) 139 [hep-ex/1001.3221]. 